\title{
Carrapatos (Acari: Ixodidae) em mamíferos silvestres do Parque Nacional da Serra da Canastra e arredores, Minas Gerais, Brasil
}

\author{
Ticks (Acari: Ixodidae) on wild mammals in the Serra da Canastra National Park and \\ surrounding areas, Minas Gerais, Brazil \\ Thiago Fernandes Martins ${ }^{\mathrm{I}^{*}}$ Ricardo Corassa Arrais, II, III \\ Fabiana Lopes Rocha ${ }^{\text {III, IV,V Jean Pierre Santos }}{ }^{\text {III }}$ Joares Adenilson May Júnior ${ }^{\mathrm{I}}$ III \\ Fernanda Cavalcanti de Azevedo ${ }^{\text {II }}$ Rogério Cunha de Paula ${ }^{\text {III, VI }}$ \\ Ronaldo Gonçalves Morato ${ }^{\text {III, VI }}$ Flávio Henrique Guimarães Rodrigues ${ }^{\text {III, VII }}$
}

Marcelo Bahia Labruna ${ }^{I}$

\section{- NOTA -}

RESUMO

O presente estudo relata ixodideos em mamiferos silvestres no Parque Nacional da Serra da Canastra e arredores, no estado de Minas Gerais, Brasil. De julho de 2005 a junho de 2014, foram coletadas 58 larvas, 133 ninfas e 43 carrapatos adultos em 40 eventos de captura de mamíferos de vida livre do Parque e arredores. Nos hospedeiros da ordem Carnivora, foram identificados Amblyomma ovale Koch, 1844, Amblyomma sculptum Berlese, 1888, Amblyomma tigrinum Koch, 1844, Dermacentor nitens Neumann, 1897 e Rhipicephalus microplus (Canestrini, 1888). Em hospedeiros da ordem Xenarthra, foram identificados espécimes de Amblyomma pseudoconcolor Aragão, 1908 e A. sculptum. Este estudo relata os primeiros registros dos ixodideos D. nitens e R. microplus parasitando Lycalopex vetulus, e A. ovale em Leopardus tigrinus no pais. Os achados do presente estudo indicam a proximidade entre os animais domésticos e silvestres causada pela expansão agropecuária no território brasileiro.

Palavras-chave: carrapatos, mamíferos silvestres, Parque Nacional da Serra da Canastra, Minas Gerais, Brasil.

\section{ABSTRACT}

This research reports ticks on wild mammals from the Serra da Canastra National Park and surrounding areas, in Minas Gerais state, Brazil. From July 2005 to June 2014, 58 larvae, 133 nymphs and 43 adult ticks were collected in 40 capture events of free-living mammals at the Park and surrounding areas. The tick species Amblyomma ovale Koch, 1844, Amblyomma sculptum Berlese, 1888, Amblyomma tigrinum Koch, 1844, Dermacentor nitens Neumann, 1897, and Rhipicephalus microplus (Canestrini, 1888) were identified on hosts of the order Carnivora. Specimens of Amblyomma pseudoconcolor Aragão, 1908 and A. sculptum were identified on the hosts of the order Xenarthra. We provide the first records of $\boldsymbol{D}$. nitens and $\boldsymbol{R}$. microplus ticks parasitizing Lycalopex vetulus, and A. ovale on Leopardus tigrinus in Brazil. The findings of this research indicate the sharing of environment by domestic and wild animals caused by agricultural expansion in the Brazilian landscape.

Key words: ticks, wild mammals, Serra da Canastra National Park, Minas Gerais, Brazil.

Os carrapatos são artrópodes ectoparasitas da classe Arachnida, de distribuição mundial, que parasitam vertebrados terrestres, anfíbios, répteis, aves e mamíferos (ARAGÃO, 1936). Dadas as particularidades de seus hábitos alimentares, os carrapatos constituem o primeiro grupo em importância de vetores de doenças infecciosas para animais e o segundo para humanos. Entre os microrganismos transmitidos, incluem-se vírus, bactérias, protozoários e helmintos (BARROSBATTESTI et al., 2006). As espécies de carrapatos que parasitam animais domésticos são as mais

\footnotetext{
IDepartamento de Medicina Veterinária Preventiva e Saúde Animal, Faculdade de Medicina Veterinária e Zootecnia, Universidade de São Paulo (USP), Av. Prof. Orlando Marques de Paiva, 87, Cidade Universitária, 05508-000, São Paulo, SP, Brasil. E-mail: thiagodogo@hotmail.com.*Autor para correspondência.

IIPrograma de Conservação Mamíferos do Cerrado, Araguari, MG, Brasil.

III Instituto Pró-Carnívoros, Atibaia, SP, Brasil.

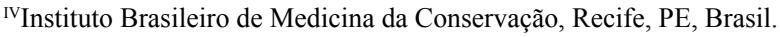

vPrograma de Pós- graduação em biologia parasitária, Instituto Oswaldo Cruz (IOC), Rio de Janeiro, RJ, Brasil.

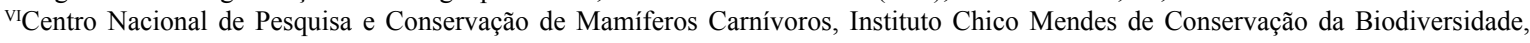
Atibaia, SP, Brasil.

${ }^{\mathrm{VII}}$ Universidade Federal de Minas Gerais (UFMG), Belo Horizonte, MG, Brasil.
} 
estudadas, porém, as espécies que parasitam a fauna silvestre ainda são pouco conhecidas, principalmente em relação à sua taxonomia, biologia, ecologia, distribuição geográfica, hospedeiros habituais e capacidade vetorial de bioagentes (BARROSBATTESTI et al., 2006).

O Parque Nacional da Serra da Canastra (PNSC) possui uma área de 200 mil hectares e está localizado no sudoeste do estado de Minas Gerais, Brasil. É composto por várias fitofisionomias do bioma Cerrado e representa uma das principais Unidades de Conservação deste bioma. O Parque abriga e protege inúmeras espécies da flora e da fauna do Cerrado, incluindo espécies raras, endêmicas e ameaçadas de extinção (BIZERRIL et al., 2008; GAMBARINI et al., 2011).

O Cerrado, segundo maior bioma brasileiro, é considerado um hotspot de biodiversidade, com aproximadamente $80 \%$ de sua área degradada pela ação antrópica, principalmente devido às atividades agrícolas (MYERS et al., 2000; SILVA et al., 2006). Esta contínua ocupação leva a uma maior proximidade da fauna silvestre com animais domésticos, possivelmente levando a um intercâmbio de parasitas entre eles. Devido à importância do conhecimento da ixodofauna parasita de animais silvestres in situ, o objetivo deste trabalho foi relatar a ocorrência de carrapatos em mamíferos silvestres no PNSC e arredores.

Os carrapatos dos mamíferos de vida livre foram coletados em 40 eventos de captura, entre julho de 2005 e junho de 2014, no PNSC e no seu entorno. Os animais foram capturados como parte de um programa de conservação da biodiversidade regional, incluindo educação ambiental, biologia comportamental, ecologia, saúde, epidemiologia, genética e reprodução de carnívoros silvestres.

Ao todo, foram capturados 40 mamíferos, sendo 17 indivíduos de Lycalopex vetulus, 11 Cerdocyon thous, três Leopardus pardalis, dois Puma concolor, dois Leopardus tigrinus, dois Conepatus semistriatus, um Lontra longicaudis, um Myrmecophaga tridactyla e um Euphractus sexcinctus. Os animais amostrados pertenciam a diferentes faixas etárias e sexos.

Os ixodídeos fixados aos mamíferos silvestres foram detectados na pele dos animais por inspeção e removidos manualmente. Os exemplares coletados foram armazenados em álcool $70 \%$ até a sua identificação no Laboratório de Doenças Parasitárias da Faculdade de Medicina Veterinária e Zootecnia da Universidade de São Paulo (FMVZUSP), com auxílio de estereomicroscópio com iluminação incidente, chaves taxonômicas e literatura correspondente (BARROS-BATTESTI et al., 2006; MARTINS et al., 2010; NAVA et al., 2014). Larvas do gênero Amblyomma não puderam ser identificadas em nível de espécie, pois não há literatura completa disponível. Todos os ixodídeos foram depositados na Coleção Nacional de Carrapatos (CNC) da FMVZUSP, sob os números de acesso: CNC-979, CNC980, CNC-1022, CNC-2550, CNC-2551, CNC-2552, CNC-2553, CNC-2554, CNC-2825.

Ao todo, foram identificados 58 larvas, 133 ninfas e 43 adultos, totalizando 234 exemplares de seis espécies distintas de carrapatos. As identificações dos ixodídeos segundo as espécies de hospedeiros estão apresentadas na tabela 1 . Dentre os 40 mamíferos inspecionados, não foram encontrados carrapatos em sete $\boldsymbol{L}$. vetulus, cinco $\boldsymbol{C}$. thous, três $\boldsymbol{L}$. pardalis, dois $C$. semistriatus, um $P$. concolor e um L. tigrinus. Das associações carrapato-hospedeiro encontradas, destacam-se os relatos, inéditos no país, de Dermacentor nitens Neumann, 1897, e Rhipicephalus microplus (Canestrini, 1888) em L. vetulus e Amblyomma ovale Koch, 1844, em $\boldsymbol{L}$. tigrinus.

A espécie D. nitens parasita primariamente equídeos, enquanto $\boldsymbol{R}$. microplus, bovídeos domésticos (BARROS-BATTESTI et al., 2006). A identificação destas duas espécies de ixodídeos parasitando $\boldsymbol{L}$. vetulus, assim como $\boldsymbol{R}$. microplus em C. thous e $\boldsymbol{P}$. concolor pode indicar a proximidade entre animais domésticos e silvestres causada pela expansão agropecuária na área do estudo. Há apenas um relato de $\boldsymbol{D}$. nitens em canídeo silvestre no território nacional, sendo este em $\boldsymbol{C}$. thous; já para $\boldsymbol{R}$. microplus existem relatos em Chrysocyon brachyurus e $\boldsymbol{C}$. thous (LABRUNA et al., 2005). O presente registro de D. nitens e $\boldsymbol{R}$. microplus parasitando $\boldsymbol{L}$. vetulus reforça o achado prévio em literatura destas duas espécies de carrapatos utilizarem canídeos silvestres como hospedeiros.

O estágio adulto do carrapato $A$. ovale é amplamente encontrado parasitando carnívoros silvestres, como pode ser observado nos resultados do presente estudo, corroborando desta forma com LABRUNA et al. (2005), que indicaram $A$. ovale como a espécie de ixodídeo mais frequentemente coletada em carnívoros silvestres no Brasil. Por outro lado, a distribuição geográfica de $\boldsymbol{A}$. ovale vai além do bioma Cerrado, estando presente na Amazônia, Mata Atlântica e Pantanal, onde o estágio adulto parasita principalmente cães domésticos e Tapirus terrestris (GUGLIELMONE et al., 2003; LABRUNA et al., 2005; LABRUNA \& GUGLIELMONE, 2009). 
Tabela 1 - Carrapatos coletados em mamíferos silvestres de vida livre no Parque Nacional da Serra da Canastra e arredores, estado de Minas Gerais, Brasil, de julho de 2005 a junho de 2014.

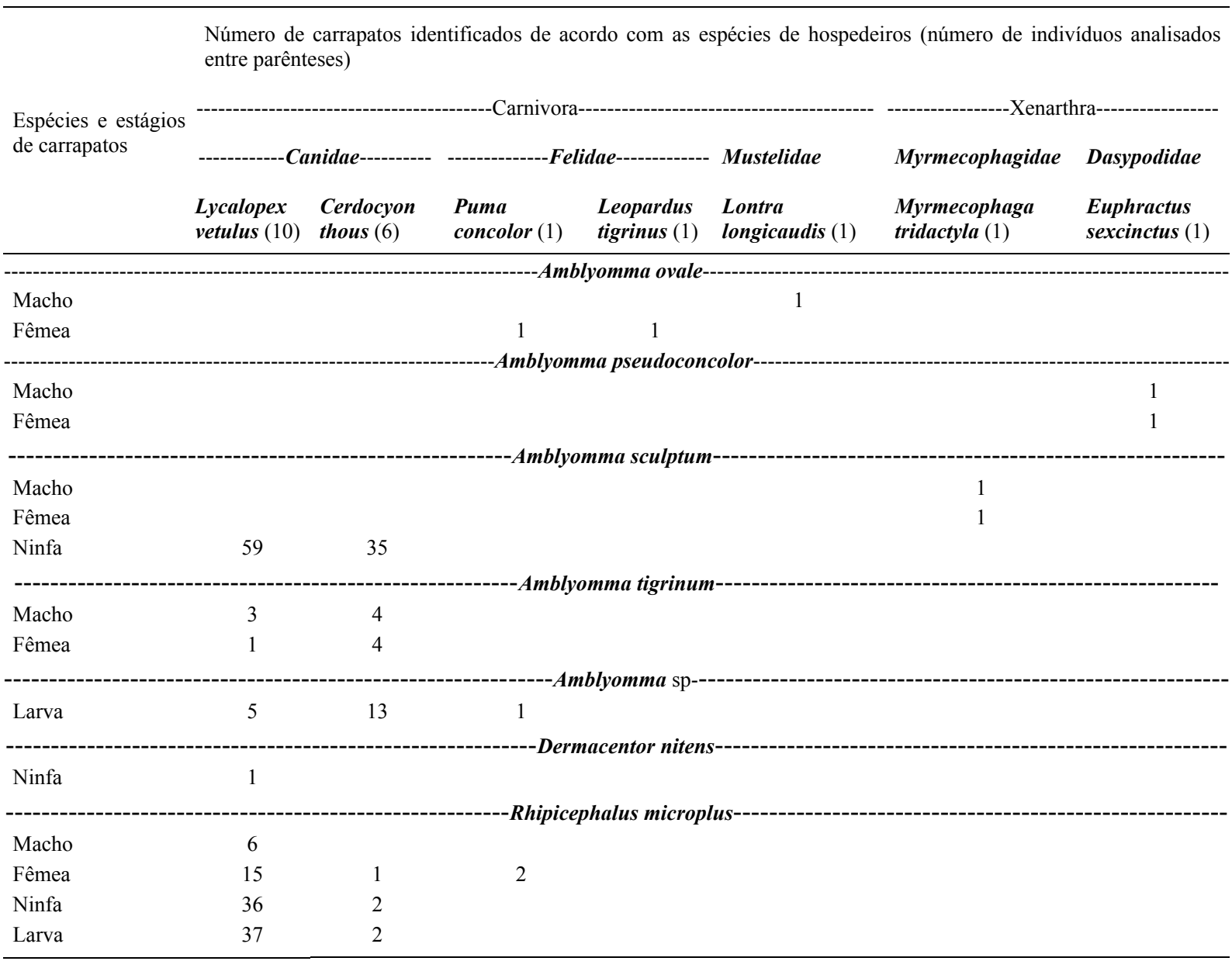

Neste trabalho, destacam-se também um grande número de amostras coletadas de canídeos silvestres, sendo elas compostas por ninfas de Amblyomma sculptum Berlese, 1888. Esta espécie pertence ao complexo Amblyomma cajennense (Fabricius, 1787), que recentemente foi desmembrado em seis espécies válidas, dentre elas, $\boldsymbol{A}$. sculptum como único representante na região Sudeste do Brasil (NAVA et al., 2014). Os resultados do presente trabalho indicam uma forte associação carrapatohospedeiro existente entre ninfas de $\boldsymbol{A}$. sculptum e canídeos silvestres, estando de acordo com estudos prévios de LABRUNA et al. (2005) e MARTINS et al. (2012), que demonstraram ninfas desta espécie de ixodídeo, publicado como $A$. cajennense, frequentemente encontradas em canídeos silvestres provenientes de várias regiões do país. As demais associações carrapato-hospedeiro (Tabela 1) foram previamente relatadas em diferentes trabalhos em diversos locais do território nacional, inclusive no bioma Cerrado, incluindo trabalhos que amostraram o próprio PNSC (BOTELHO et al., 1989; BARROS \& BAGGIO, 1992; EVANS et al., 2000; LABRUNA et al., 2005; MARTINS et al., 2012).

De acordo com as infestações encontradas, os mamíferos silvestres amostrados no presente estudo apresentaram diversidade na ixodofauna. Estes resultados são importantes, não apenas para entender o papel das espécies silvestres na manutenção das populações de carrapatos, como também para monitorar a saúde das populações de animais silvestres, principalmente em áreas naturais de constante pressão antrópica, como no caso do Cerrado brasileiro.

\section{COMITÊ DE ÉTICA E BIOSSEGURANÇA}

Protocolado sob o nº 2510/2011 da Universidade de São Paulo (USP), Faculdade de Medicina Veterinária e Zootecnia (FMVZ). 


\section{AGRADECIMENTOS}

O presente trabalho recebeu auxílio financeiro da Fundação de Amparo à Pesquisa do Estado de São Paulo (FAPESP) e Conselho Nacional de Desenvolvimento Científico e Tecnológico $(\mathrm{CNPq})$ para identificação dos carrapatos. Agradecemos também ao Instituto Pró-Carnívoros (IPC), ao Instituto Chico Mendes de Conservação da Biodiversidade (ICMBio), Centro Nacional de Pesquisa e Conservação de Mamíferos Carnívoros (CENAP), Fundo Nacional do Meio Ambiente (FNMA) e Wildlife Conservation Society (WCS).

\section{REFERÊNCIAS}

ARAGÃO, H.B. Ixodidas brasileiros e de alguns países limitrophes. Memórias do Instituto Oswaldo Cruz, v.31, n.4, p.759-843, 1936.

BARROS-BATTESTI, D.M. et. al. Carrapatos de importância médico-veterinária da região neotropical: um guia ilustrado para identificação de espécies. São Paulo: Vox/ICTTD-3/Butantan, 2006. 223p.

BARROS, D.M.; BAGGIO, D. Ectoparasites Ixodida Leach, 1817 on wild mammals in the State of Paraná, Brazil. Memórias do Instituto Oswaldo Cruz, v.87, n.2, p.291-296, 1992.

BIZERRIL, M. et. al. Um lugar chamado Canastra. Atibaia: CENAP/ICMBio e Instituto Pró-Carnívoros, 2008. 79p.

BOTELHO, J.R. et. al. Interrelações entre Acari Ixodidae e hospedeiros Edentata da Serra da Canastra, Minas Gerais, Brasil. Memórias do Instituto Oswaldo Cruz, v.84, n.1, p.61-64, 1989.

EVANS, D.E. et. al. A review of the ticks (Acari: Ixodida) of Brazil, their hosts and geographic distribution - 1. The State of Rio Grande do Sul, Southern Brazil. Memórias do Instituto Oswaldo Cruz, v.95, n.4, p.453-470, 2000.

GAMBARINI, A. et. al. Serra da Canastra: diversidade infinita. São Paulo, 2011. 240p.
GUGLIELMONE, A.A. et. al. Amblyomma aureolatum (Pallas, 1772) and Amblyomma ovale Kock, 1844: hosts, distribution and 16S rDNA sequences. Veterinary Parasitology, v.113, n.34, p.273-288, 2003. Disponível em: <http://dx.doi.org/10.1016/ S0304-4017(03)00083-9>. Acesso em: 13 nov. 2013.

LABRUNA, M.B.; GUGLIELMONE, A.A. Ticks of new world tapirs. Tapir Conservation, v.18, n.25, p.21-28, 2009.

LABRUNA, M.B. et. al. Ticks (Acari: Ixodida) on wild carnivores in Brazil. Experimental and Applied Acarology, v.36, n.1-2, p.149-163, 2005. Disponível em: <http://dx.doi.org/10.1007/ s10493-005-2563-1>. Acesso em: 13 dez. 2013.

MARTINS, T.F. et al.m Ticks on free-living wild mammals in Emas National Park, Goiás State, central Brazil. Systematic and Applied Acarology, v.16, n.3, p.201-206, 2012.

MARTINS, T.F. et al. Nymphs of the genus Amblyomma (Acari: Ixodidae) of Brazil: descriptions, redescriptions, and identification key. Ticks and Tick-borne Diseases, v.1, n.2, p.75-99, 2010. Disponível em: <http://dx.doi.org/10.1016/j.ttbdis.2010.03.002>. Acesso em: 26 maio 2013 .

MYERS, N. et. al. Biodiversity hotspots for conservation priorities. Nature, v.403, n.6772, p.853-858, 2000. Disponível em: <http:// dx.doi.org/10.1038/35002501>. Acesso em: 24 jan. 2014.

NAVA, S. et. al. Reassessment of the taxonomic status of Amblyomma cajennense (Fabricius, 1787) with the description of three new species, Amblyomma tonelliae n. sp., Amblyomma interandinum n. sp. and Amblyomma patinoi n. sp., and reinstatement of Amblyomma mixtum Koch, 1844 and Amblyomma sculptum Berlese, 1888 (Ixodida: Ixodidae). Ticks and Tick-borne Diseases, v.5, n.3, p.252-276, 2014. Disponível em: <http://dx.doi.org/10.1016/j.ttbdis.2013.11.004>. Acesso em: 14 abr. 2014.

SILVA, J.F. et. al. Spatial heterogeneity, land use and conservation in the Cerrado region of Brazil. Journal of Biogeography, v.33, n.3, p.536-548, 2006. Disponível em: <http://dx.doi.org/10.1111 /j.1365-2699.2005.01422.x>. Acesso em: 11 fev. 2014 